\title{
Aploplejía hipofisaria como presentación de adenoma hipofisario
}

\section{Pituitary apoplexy as presentation of pituitary adenoma}

\author{
Víctor Alonso Vargas-Rubio', \\ Javier Arias-Stella Castillo²
}

\author{
Vargas-Rubio V.Arias-Stella C J.Aploplejía hipofisaria como presentación \\ de adenoma hipofisario. Rev Soc Peru Med Interna. 2021;34(3): I I4-I I 8. \\ https://doi.org/10.36393/spmi.v34i3.612
}

\begin{abstract}
RESUMEN
Mujer de 55 años que acudió por cefalea súbita intensa, ptosis palpebral izquierda asociada a disminución de la agudeza visual a predominio del ojo izquierdo; además, los exámenes de laboratorio evidenciaron hiponatremia más hipopituitarismo. La resonancia magnética cerebral mostró un adenoma pituitario con gran componente necrótico que posteriormente fue confirmado en el estudio anatomo patológico. Se concluyó en una apoplejía hipofisaria como forma de presentación de un adenoma hipofisario sin diagnóstico previo, con buena evolución de los síntomas visuales después de la cirugía.
\end{abstract}

Palabras claves:Apoplejía hipofisaria, adenoma hipofisario, tumor hipofisario, cefalea.

\section{ABSTRACT}

A 55-year-old woman who attended for sudden intense headache, left palpebral ptosis associated with decreased visual acuity to predominance of the left eye; in addition, laboratory tests showed hyponatremia plus hypopituitarism. The cerebral magnetic resonance showed a pituitary adenoma with a large necrotic component that was later confirmed in the pathological study. It was concluded in a pituitary apoplexy as a form of presentation of a pituitary adenoma without prior diagnosis, with good evolution of visual symptoms after surgery.

Keywords: Pituitary apoplexy, pituitary adenoma, pituitary tumor, headache.

\section{INTRODUCCIÓN}

La apoplejía hipofisaria es una patología aguda potencialmente mortal cuyas manifestaciones clínicas están dadas por la expansión brusca de la hipófisis, generalmente supraselar y paraselar, secundaria a un infarto isquémico

Médico internista. Facultad de Medicina, Universidad Peruana Cayetano Heredia. Servicio de Emergencia, Hospital Nacional Arzobispo Loayza, Lima, Perú.

2 Médico patólogo. Instituto de Patología y Biología Molecular Arias Stella, Lima, Perú. o hemorrágico de un adenoma hipofisario, siendo el macroadenoma el tumor más relacionado a este cuadro. ${ }^{1}$ Esta entidad debería denominarse apoplejía tumoral hipofisaria ya que la hemorragia intrínseca dentro de la glándula pituitaria normal refleja patologías como el síndrome de Sheehan y otras patologías como metástasis o traumatismos a ese nivel. ${ }^{2}$ Además, se debe resaltar que esta definición es más clínica que patológica porque no toda hemorragia o infarto hipofisario se presentará como una apoplejía donde los síntomas resaltantes son cefalea intensa, trastornos visuales e hipopituitarismo. ${ }^{3}$ 
Presentamos el caso de una paciente sin antecedente de patología hipofisaria y con un cuadro clínico sugestivo de esta entidad y posteriormente se pudo confirmar el diagnóstico con estudio anatomo patológico.

\section{PRESENTACIÓN DEL CASO}

Mujer de 55 años de edad, procedente de Los Olivos, Lima, con antecedente de diabetes mellitus tipo 2 hace ocho años con tratamiento metformina y vildagliptina, aparentemente controlada; además, hipertensión arterial hace 5 años con tratamiento irbesartán e hidroclorotiazida, aparentemente controlada. Niega hábitos nocivos. Acude al Servicio de Emergencia del Hospital Nacional Arzobispo Loayza con un tiempo de enfermedad de cuatro días, de inicio súbito y curso progresivo, refiriendo que inició con cefalea holocraneana tipo punzada, de intensidad 9/10, asociado a náuseas y vómitos por lo que acudió a un centro particular donde le recetaron analgésicos y los síntomas remitieron parcialmente. Tres días antes se agrega "caída del párpado superior izquierdo", continúa con sintomáticos. Un día antes del ingreso presentó somnolencia por lo que acudió a centro particular donde le solicitaron estudios de imágenes ante la posibilidad de un "infarto cerebral", se le realizaron estudios y decidió acudir al hospital.

Al examen físico, presión arterial de 110/70 mm Hg, frecuencia cardiaca de 82 latidos $/ \mathrm{min}$, frecuencia respiratoria de 20 excursiones/min y $\mathrm{SatO}_{2} \quad 98 \%$. Somnolienta, orientada en persona mas no en tiempo y lugar, ptosis palpebral izquierda asociada a midriasis arreactiva (Imagen 1 y 2), ceguera en el ojo izquierdo y disminución de agudeza visual en ojo derecho, no se pudo evaluar campimetría por estado de conciencia, fuerza muscular 4/5 en las cuatro extremidades, signo de Babinsky $(+)$ bilateral, no signos meníngeos.

De los exámenes de laboratorio, lo resaltante fue un sodio de $111 \mathrm{mEq} / \mathrm{L}$; y, el hemograma, urea, creatinina, glicemia y albúmina estuvieron dentro de los rangos normales.

Tomografía cerebral sin contraste: no se evidenció signos isquémicos ni hemorrágicos. Resonancia magnética cerebral: se evidenció un proceso expansivo intra, supra, infra $y$ paraselar izquierdo, de naturaleza mixta con predominio de componente de aspecto necrótico, que

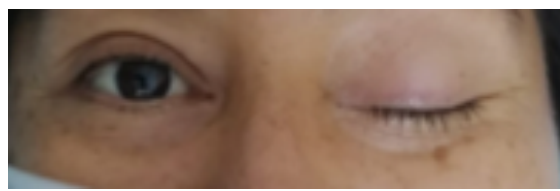

Figura I

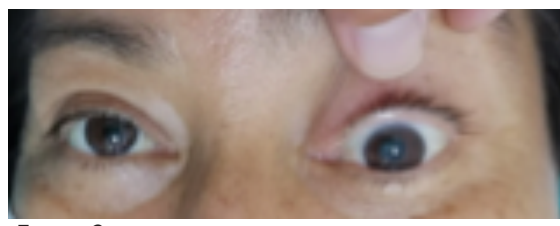

Figura 2

Figuras I - 2. Ptosis palpebral izquierda asociada a midriasis arreactiva.

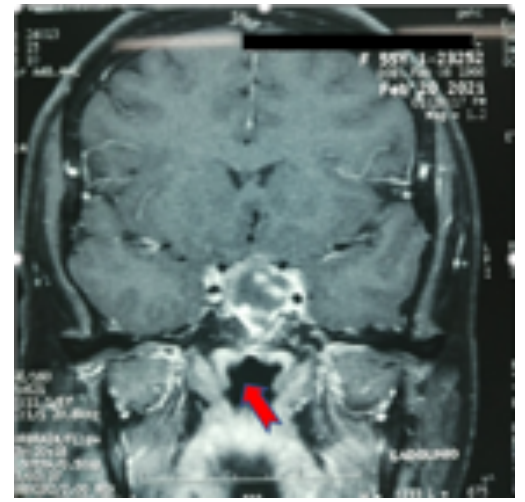

Figura 3

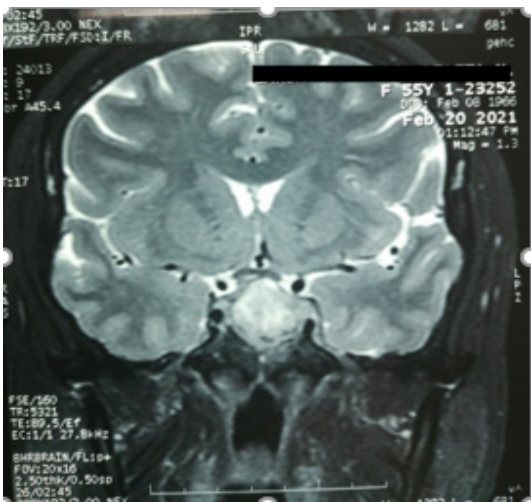

Figura 4

Figuras 3 - 4. RMN cerebral en secuencia TI con contraste (Fig. 3) y en secuencia T2 (Fig.4): masa intra, supra, infra y paraselar, heterogénea, que comprime estructuras vecinas, además engrosamiento de la mucosa del seno esfenoidal (Flecha roja).

condicionaba una compresión del quiasma óptico y en su extensión paraselar izquierda comprometía el seno cavernoso, comprimiendo el trayecto intracanalicular del III nervio craneal izquierdo; además, engrosamiento mucoso marginal de las celdillas etmoidales y de la mucosa del seno esfenoidal.

Ante la sospecha de apoplejía hipofisaria se solicitó un panel hormonal y se inició terapia corticoide endovenoso, la muestra que se obtuvo en el siguiente turno mostró TSH 0,5 (VN 0,5-5 $\mu \mathrm{UI} / \mathrm{ml})$, T4 L 1,45 (VN 0,9-1,7 ng/dl), cortisol AM basal12,89 $\mu \mathrm{g} / \mathrm{dl}$ (VN 6,2-19,4 $\mu \mathrm{g} / \mathrm{dl})$, ACTH basal 62,8 pg/ml (VN 5-63 pg/ml), FSH 6,3 mUI/ml (VN 48-163 mUI/ml), LH 1,1 mUI/ml (VN 15,2-77,5 mUI/ml), prolactina 0,8 (VN 3,4-24,1 ng/ml), IGF-1 $65 \mathrm{ng} / \mathrm{ml}$ (VN $70-280 \mathrm{ng} / \mathrm{ml})$.

Fue evaluada por el servicio de neurocirugía quienes la catalogaron como un proceso expansivo selar, probable macroadenoma hipofisiario de indicación quirúrgica con 96 horas de evolución y al momento no se contaba con capacidad resolutiva, por lo que se inició la referencia a otro centro. La paciente recibió corticoterapia endovenosa y restricción hídrica, para el día 5 de hospitalización tenía un sodio sérico de $133 \mathrm{mEq} / \mathrm{L}$ pero, por demoras en la referencia, la paciente solicitó su retiro voluntario. 
Acudió a un centro particular donde la programaron para cirugía transesfenoidal. Le extirparon varios fragmentos de tejido pardo claro, de consistencia blanda que en conjunto midieron $1,3 \times 1,1 \times 0,2 \mathrm{~cm}$, siendo el reporte de patología quirúrgica de un adenoma hipofisiario con extensa necrosis tipo infarto, consistente con apoplejía hipofisaria (Figura $5)$.

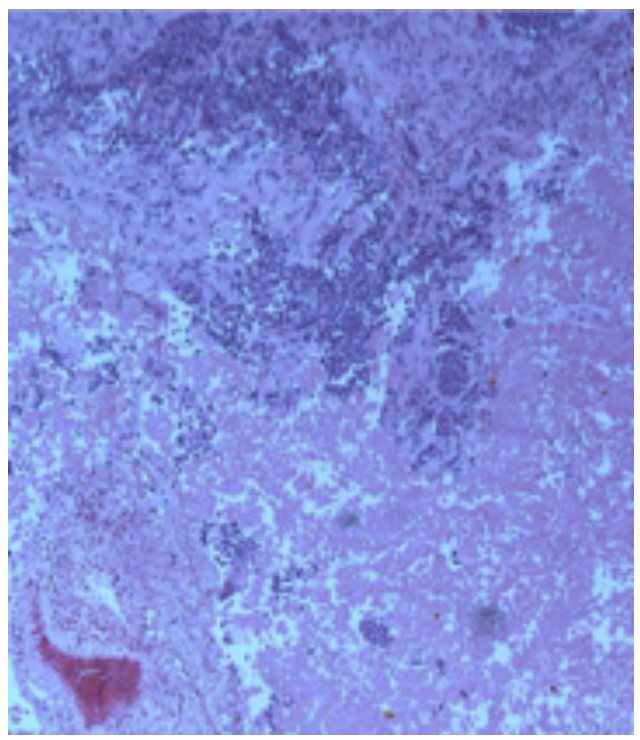

Figura 5. Anatomía patológica de un adenoma hipofisario con necrosis tipo infarto.

\section{DISCUSIÓN}

La apoplejía hipofisaria es una entidad rara, descrita por primera vez por Bailey en 1898, donde describió el caso de una hemorragia asociada a una tumoración de hipófisis. Pero fue hasta 1950 donde se acuñó el término de "apoplejía pituitaria" por Brougham. ${ }^{4}$ Aunque, actualmente se tiene conocimiento de que esta patología fue descrita en la Colección Hipocrática del siglo $\mathrm{V}$ antes de nuestra era. ${ }^{5}$ La prevalencia de esta enfermedad es de 6,2 casos por 100 000 habitantes $^{6}$, teniendo una incidencia de 0,17 casos por 100000 personas/año. ${ }^{7}$ Se presenta entre 2 a $12 \%$ de pacientes con adenoma hipofisario, siendo el adenoma no funcionante el tipo más frecuente. En el $80 \%$ de casos la apoplejía hipofisaria es la primera manifestación de esta tumoración hipofisaria benigna. ${ }^{8,9}$ Es más frecuente en varones con una relación 2:1 sobre el sexo femenino, y suele presentarse entre la quinta y sexta década de vida. ${ }^{10} \mathrm{En}$ nuestro caso, la paciente no tenía antecedente de patología pituitaria previa, por lo que la apoplejía hipofisaria fue la forma de presentación del adenoma hipofisario; además, el adenoma era de tipo no funcionante y la paciente se encontraba en el rango de edad donde esta entidad se presenta con mayor frecuencia.

Se han descrito diversos factores de riesgo para desarrollar esta condición, dentro de los más importantes tenemos a la hipertensión arterial, que es la más frecuente, la anticoagulación y las cirugías mayores, en especial el bypass coronario por el riesgo de hipotensión y la variabilidad de presión arterial durante el acto operatorio; otros factores descritos son el embarazo, traumatismo craneal, inicio o suspensión de terapia con agonistas dopaminérgicos, tests dinámicos de función hipofisaria con GnRH, TRH y $\mathrm{CRH}$, radioterapia, coagulopatías y uso de estrógeno. ${ }^{11}$ Cabe recalcar que estos factores de riesgo se encuentran hasta en un $40 \%$ de los casos. ${ }^{11,12}$ Con respecto a nuestro caso, el único factor de riesgo que se pudo identificar fue la hipertensión arterial, aunque esta aparentemente estuvo controlada. Sin embargo, se debe recordar que en más de la mitad de los casos no se logra identificar algún factor de riesgo que precipite el desarrollo de esta patología.

Dentro de la fisiopatología de esta entidad tenemos que al tratarse generalmente de macroadenomas, estos son propensos a presentar hemorragia o isquemia, ya que superan el suministro de sangre aportados por los vasos sanguíneos que llegan a ese nivel o porque esta masa comprime los vasos hipofisarios superiores contra el diafragma selar. También, estos tumores van a presentar vasos sanguíneos frágiles, con una maduración incompleta y escasas fenestraciones que explicarían la tendencia a la hemorragia; así, todo esto conlleva a un aumento de la presión intraselar, que a su vez produce compresión de estructuras vecinas, lo que explicaría la cefalea y la oftalmoplejía, además, el por qué algunos macroadenomas hipofisarios hemorrágicos o necróticos no presentan síntomas. ${ }^{13}$

Con respecto al componente hormonal, en la fase aguda el dosaje de hormonas hipofisarias en sangre puede resultar normal y conforme pasen los días a meses evolucionar en panhipopituitarismo, que puede ser transitorio o permanente, y silla turca vacía secundaria, con o sin deficiencias hormonales. ${ }^{14}$

La manifestación clínica más frecuente es la cefalea (9097\%), de inicio súbito, gran intensidad, mayormente de localización retroorbitaria pero puede ser bifrontal, suboccipital o de localización difusa; luego, son las alteraciones visuales, ya sea por parálisis de nervios oculomotores (40-70\%), donde el tercer par craneal es afectado con mayor frecuencia, disminución de agudeza visual (41-56\%) o por defectos en los campos visuales (47-75\%), siendo la hemianopsia bitemporal la más característica. ${ }^{15}$ Otros síntomas inespecíficos son náuseas y vómitos (69-80\%); disminución del nivel de conciencia (13\%) debido probablemente al incremento de la presión intracraneal, compromiso hipotalámico, trastorno endocrino $\mathrm{y} / \mathrm{o}$ trastorno hidroelectrolítico asociado; $\mathrm{y}$, signos meníngeos, por extravasación de sangre e irritación meníngea. ${ }^{16}$ El déficit hormonal adenohipofisario se presenta hasta en un $80 \%$, las hormonas más comprometidas son gonadotrofinas (75\%), TSH (50\%) y ACTH (70\%), siendo esta última potencialmente fatal; $\mathrm{y}$, los pacientes que tienen niveles bajos de prolactina sérica al momento de la presentación tienen una presión intraselar mayor y menos probabilidades de recuperar la función hormonal después de la cirugía. ${ }^{17}$

También se observa hiponatremia hasta en el $40 \%$ de los casos y esta es secundaria a síndrome de secreción 
inadecuada de hormona antidiurética, hipocortisolismo e hipotiroidismo. ${ }^{18}$

El diagnóstico se realiza teniendo las manifestaciones clínicas y la presencia de un adenoma hipofisario previamente ya conocido o recientemente descubierto, por lo tanto, es importante el estudio de imagen; La tomografía cerebral sin contraste es de mucha ayuda en los casos agudos (24 a 48 horas), ya que se puede observar el componente hemorrágico, pasado ese tiempo la intensidad de la sangre disminuye debido a la degradación de la hemoglobina; $\mathrm{y}$, es por esto que este estudio de imagen no diagnostica el $44 \%$ de los casos. ${ }^{19}$ La resonancia magnética cerebral tiene una sensibilidad mayor (88-90\%) para diagnóstico de esta patología, las características dependen del tiempo de evolución, así tenemos, que en la fase aguda (0-7 días), encontraremos hipointensidad en secuencias T1 y T2: en la fase subaguda (7-21 días), hiperintensidad en secuencias T1 y T2 y; y, por último, en la fase crónica (>21 días), hipointenisad en ambas secuencias. ${ }^{20}$

El engrosamiento de la mucosa del seno esfenoidal es un hallazgo importante en la resonancia magnética cerebral que se describe en relación a apoplejía hipofisaria y que probablemente se deba a congestión venosa en esta región. ${ }^{20,}{ }^{21}$ Con respecto a nuestra paciente. podemos determinar que radiológicamente su cuadro corresponde a una fase subaguda de la enfermedad ya que en la secuencia T2 se aprecian áreas de hipo e hiperintensidad y, además, se pudo observar el engrosamiento de la mucosa del seno esfenoidal.

El tratamiento de la apoplejía hipofisaria se basa en primer lugar en estabilizar al paciente, esto incluye reemplazo hormonal, siendo el eje adrenal el más importante, la hidrocortisona es el corticoide de elección, y si la paciente presentara panhipopituitarismo debemos empezar el reemplazo de cortisol y retrasar por 24 horas el reemplazo tiroideo, ya que al iniciar el tratamiento con levotiroxina primero la tasa metabólica del paciente aumentará con un eje adrenal que no podrá responder ante esta situación y podría desencadenarse una crisis adrenal; también, debemos corregir la hiponatremia que se presenta en casi la mitad de los casos. ${ }^{22}$

La indicación de tratamiento quirúrgico de emergencia se da en aquellos pacientes con apoplejía hipofisaria aguda, es decir, en aquellos que presenten alteraciones visuales con un tiempo de enfermedad de 24 a 72 horas, siendo los objetivos de esta intervención la descompresión de las estructuras implicadas en la aparición de los síntomas, evacuación del hematoma, resección del tumor o quiste y reparar el defecto de base de cráneo si es que lo hubiera; en los casos de apoplejía hipofisaria subaguda ( $>72$ horas de evolución) el tratamiento quirúrgico está indicado en aquellos pacientes que tienen alto riesgo de empeorar, como los que presentan una gran hemorragia, uso de anticoagulación, coagulopatías, pérdida de visión severa o pacientes con tumores invasivos; el objetivo en este caso se centra en la máxima resección del tumor. ${ }^{23}$

Con respecto al pronóstico, se ha visto que tanto el tratamiento conservador como quirúrgico (descompresión) pueden llevar a una recuperación endocrina y visual. ${ }^{24} \mathrm{Sin}$ embargo, se ha visto mejores resultados con las indicaciones quirúrgicas descritas en el párrafo anterior.

En el seguimiento de nuestra paciente a cuatro meses, se observó una mejoría parcial del déficit visual, con mejoría de la agudeza visual y elevación del párpado superior izquierdo, a pesar de que el tiempo transcurrido entre el diagnóstico y la cirugía fue de 30 días. Se han reportado casos con un tiempo de espera similar, en la cual no hubo mejoría del déficit visual. ${ }^{25}$ Esto se podría deber al uso de corticoides, ya que en nuestro caso lo usamos desde el momento del diagnóstico, mientras que en el reporte lo usaron en el perioperatorio. Con respecto a la parte hormonal, la paciente actualmente se encuentra con tratamiento sustitutivo adrenal y tiroideo.

En conclusión, ante un paciente con cefalea intensa y alteraciones visuales, ya sea disminución de la agudeza visual, defecto en la campimetría o trastorno oculomotor debemos sospechar en apoplejía hipofisaria, ampliando el estudio con dosaje hormonal y resonancia magnética cerebral.

\section{REFERENCIAS BIBLIOGRÁFICAS}

I. Carral San Laureano F, Gavilan Villarejo I, Garcia Gil D, et al. Apoplejía hipofisaria. Protocolo de actuación diagnóstica y terapéutica en urgencias. Emergencias. 200I; I3:279-282.

2. Bi WL, Dunn IF, Laws ER Jr. Pituitary apoplexy. Endocrine. 2015 Feb;48(I):69-75. doi: I0.1007/s I 2020-0I4-0359-y. Epub 2014 Jul 26. PMID: 25063308.

3. Robles Torres E, Mendoza Choqueticlla JA, Bartolomé Copa JL, Leal Curi L \& Carrasco Palomeque I. (20I7).Apoplejía hipofisaria, presentación de dos casos clínicos y revisión del tema. Revista Cubana de Endocrinología, 28(3), I-I3.

4. Glezer A, Bronstein MD. Pituitary apoplexy: pathophysiology, diagnosis and management. Arch Endocrinol Metab. 2015 Jun;59(3):259-64. doi: I0.1590/2359-3997000000047. PMID: 26154095 .

5. Adams W.A case report from the ancient past. Am J Case Rep, 20I9; 20: 1907-1914.

6. Fernandez A, Karavitaki N,Wass J. Prevalence of pituitary adenomas: a community -based, cross -sectional study in Banbury (Oxfordshire, UK). Clinical Endocrinology. 2010;72, 377-382.

7. Raappana A, Koivukangas J, Ebeling T, et al, Incidence of pituitary adenomas in Northern Finland in 1992-2007.J. Clin Endocrinol Metab. September 2010, 95(9): 4268-4275.

8. Briet C, Salenave S, Chanson P. Pituitary apoplexy. Endocrinol Metab Clin North Am. 20I5 Mar;44(I): 199-209.

9. Pinto Sánchez J, Villena Chávez J, Villena Pacheco A, et al. Apoplejía pituitaria: Reporte de dos casos. Rev Med Hered. 2007; I8: I65-I72.

10. Sánchez Tinajero A., Ruiz Santillán D., Apoplejía hipofisaria secundaria a macroadenoma hipofisario, un reto diagnóstico. Rev Med Inst Mex Seguro Soc. 2019; 57(4): 259-63.

I I. Almudena V, Lecumberri B, Ángeles M. Guía de práctica clínica para el diagnóstico y tratamiento de la apoplejía hipofisaria. Endocrinol Nutr. 20I 3;60(I0):582, I- I 2.

12. Sibal L, Ball S, Connolly V, et al. Pituitary apoplexy: A review of clinical presentation, management and outcome in 45 cases. Pituitary. 2004;7:157-163.

13. Briet C, Salenave S, Bonneville J, et al. Pituitary Apoplexy. Endocrine Reviews. 2015,36(6): 622-645.

14. Catalá M, Picó A, Tortosa F, et al. Guía clínica del diagnóstico y tratamiento de la apoplejía hipofisaria. Endocrinol Nutr. 2006;53(1): 1924.

15. Wichlinska-Lubinska M, Kozera G. Pituitary apoplexy. Neurol 
Neurochir Pol. 201 9;53(6):4I 3-420

16. Pyrgelis E, Mavridis I, Meliou M. Presenting symptoms of pituitary apoplexy.J Neurol Surg A. 2018;79: 52-59.

17. Rajasekaran S., Vanderpump M., Baldeweg S., UK guidelines for the management of pituitary apoplexy. Clinical Endocrinology. 2011;74, 9-20.

18. Glezer A, Bronstein M. Pituitary apoplexy: pathophysiology, diagnosis and management. Arch Endocrinol Metab. 2015 Jun;59(3):259-64.

19. Donovan Post M, David N, Glaser J, et al. Pituitary apoplexy: Diagnosis by computed tomography. Radiology. 1980;134:665-670.

20. Boellis A, Napoli A, Romano A, et al. Pituitary apoplexy: an update on clinical and imaging features. Insights Imaging. 20I4; 5:753-762.

21. Goyal P, Utz M, Gupta N, et al., Clinical and imaging features of pituitary apoplexy and role of imaging in differentiation of clinical mimics. Quant Imaging Med Surg. 2018; 8(2): 219-231.

22. Capatina C, Inder W, Karavitaki N, et al. Management of Endocrine Disease -Pituitary tumour apoplexy. European Journal of Endocrinology. 20I5;|72: RI79-RI90.
23. Barkhoudarian G, Kelly D. Pituitary apoplexy. Neurosurg Clin N Am. 2019, I-6.

24. Goshtasbi K,Abiri A, Sahyouni R, et al.,Visual and endocrine recovery following conservative and surgical treatment of pituitary apoplexy: A meta-analysis. World Neurosurgery. 2019132: 33-40.

25. Agrawal D, Mahapatra AK.Visual outcome of blind eyes in pituitary apoplexy after transsphenoidal surgery: a series of 14 eyes. Surg Neurol. 2005 Jan;63(I):42-6; discussion 46. doi: 10.1016/j. surneu.2004.03.0 I4. PMID: I563952 I

\section{CORRESPONDENCIA:}

Víctor Alonso Vargas-Rubio

victor.vargas.rubio@upch.pe

Fecha de recepción: 19-07-2021.

Fecha de aceptación: 20-08-2021.

Conflicto de interés: ninguno, según los autores.

Financiamiento: por los autores. 